\title{
Laboratory Errors: Types and Frequency in a Tertiary Hospital in Bangladesh
}

\author{
N CHOWDHURY ${ }^{\mathrm{a}}$, M IBRAHIM ${ }^{\mathrm{b}}$, MAH KHAN ${ }^{\mathrm{c}}$
}

\section{Summary:}

Introduction: In our country, very few of clinical laboratories are running proper quality control program and to the best of our knowledge the preanalytical, analytical, and postanalytical rates of laboratory errors have not been studied extensively. In this study we evaluated the preanalytical, analytical, and postanalytical components of laboratory errors in 3,200 consecutive specimens of a clinical chemistry laboratory in a tertiary hospital for measurement of different analyte concentrations in plasma or serum. Materials and methods: This study was conducted during the period from June 2009 to July 2010 on 3,200 specimens. Analytical errors were detected by repeat analysis of primary sample and by checking quality control. Results: The numbers of preanalytical, analytical and postanalytical errors were 23, 14 and 76 respectively among 32000 tests that we have done on 3,200 specimens (average 10 tests per specimen). Moreover, the causes of errors were analyzed

\section{Introduction:}

Accurate and reliable laboratory test result is essential for proper diagnosis and treatment of the patients. Over $70 \%$ of clinical decisions are taken based on laboratory reports ${ }^{1}$. There is an increasing awareness of the importance of errors in laboratory practice and their possible negative impact on patient outcomes. Medicolegal litigations are on the rise with growing awareness amongst clients. In some cases patients filed court cases

a. Dr. Nasreen Chowdhury, Professor, Department of Biochemistry, Chattagram Maa-O-Shishu Hospital Medical College, Chittagong, Bangladesh.

b. Dr. Md. Ibrahim, Cosultant, Department of Biochemistry, Appollo Hospitals, Dhaka, Bangladesh.

c. Dr. Md. Aminul Haque Khan, Professor, Department of Biochemistry, Enam Medical College, Savar, Dhaka, Bangladesh.

Address of Correspondence: Dr. Md. Aminul Haque Khan, Professor \& Head, Department of Biochemistry, Enam Medical College, Savar, Dhaka, Bangladesh. Email: aminhkhan@yahoo.com. Phone: 01552-479546.

Received: 29 August, 2013

Accepted: 10 September, 2014 and it was found that preanalytical errors were mostly due to specimen drawn distal to IV infusion, specimen for potassium first drawn into GREY tube (containing sodium fluoride and K EDTA) and then transferred into GREEN tube, long tourniquet time and underfilling of blood collection tube. The analytical errors were due to random and systemic errors and postanalytical errors were due to transcription errors. Conclusion: Results of our study suggest that errors mostly occur in the postanalytical part of testing and they are due to transcription errors. To reduce the laboratory errors we suggest introduction of Laboratory Information System (LIS) of the clinical laboratories connected with Hospital Management System along with stringent quality control program in preanalytical, analytical and postanalytical stages.

Key words: Preanalytical, Analytical, Postanalytical, Transcription errors

(J Banagladesh Coll Phys Surg 2015; 33: 3-6)

against doctors for real or perceived reasons of dissatisfaction. Medical malpractice law suits against medical laboratories are also increasing. This has placed urgency on clinical laboratories to ensure their quality and error-free work practices to be maintained. Many strategies are used to reduce laboratory errors, including internal quality control procedures, external quality assessment programs, certification of education programs, licensing of laboratory professionals, accreditation of clinical laboratories, and the regulation of laboratory services.

A recent document from the International Organization for Standardization (ISO) recommends a new, broader definition of the term 'laboratory error' and a classification of errors according to different criteria. The more recent surveys on errors in laboratory medicine conclude that in the delivery of laboratory testing, mistakes occur more frequently before (preanalytical) and after (postanalytical) the test has been performed. Ross and Boone found that mistakes made in laboratory testing were distributed as given below: 
preanalytical $46 \%$, analytical $7 \%$, and postanalytical $47 \%^{2}$. Similar data were obtained by Bachner et al. in a CAP Q-probe study on blood bank quality-assurance practice $^{3}$ and, more recently, by Boone et al. in a survey on transfusion medicine $e^{4}$. Plebani reported that most errors are due to preanalytical factors (46-68.2\% of total errors), while a high error rate (18.5-47\% of total errors) has also been found in the post-analytical phase ${ }^{5}$.

Laboratory services have also a great influence on clinical decision making. It is reported that, $60-70 \%$ of the most important decisions on admission, discharge, and medication are based on laboratory test results ${ }^{6}$. With this high degree of influence, the quality of laboratory testing and reporting is of utmost importance. However, very few laboratories in our country are following the proper quality control program for producing better reports. Moreover, we still do not have any reference laboratory at national level. Number of technical experts, technologists, and phlebotomists are very poor in comparison to the number of growing laboratories. We are still in shortage of a well organized accreditation body in our country. In spite of all these obstacles some laboratories are trying to produce good results for their own reputation and to provide better services. We chose one of these laboratories and conducted the study for one year to detect the sources of errors in clinical laboratories. The aim of this study was to evaluate the frequency and types of mistakes in this laboratory to identify the most critical steps in the analytical testing process and to plan a corrective strategy.

\section{Materials and methods:}

The present study was carried out on 3200 specimens in the department of clinical chemistry of Apollo Hospitals, Dhaka which is a JCI accreditated 450-bed tertiary care hospital during the period July 2009 to June 2010. In clinical chemistry department around 720000 tests are done per year. On an average, 10 tests are done per specimen. The department receives specimen from OPD clinic, emergency, and IPD (Neuro ICU, Surgical ICU, Medical ICU, Neonatal ICU, CCU and wards). Blood drawing and specimen collection are performed by phlebotomists (mostly OPD), physicians and nurses from the different areas. All test requests are made directly by consultant and ward staff using the Hospital Information System (HIS), which allows the immediate printing of barcode labels for the identification of all patient specimens. Specimens are transported to laboratory manually by ward staff or patient care attendant. Currently laboratory medicine department of Apollo Hospitals, Dhaka is not supported by Laboratory Information System (LIS), so all results that have been generated after analysis are fed into the system manually by data entry operator. We have maintained specific turn around time for each parameter followed by reporting of critical value. We had a policy for stringent quality control program (internal quality control and proficiency testing/external quality control), specimen rejection criteria, with the documentation in the event log. We have detected all the errors from notification by physician and errors that have been detected in laboratory consultant desk by checking history and previous value for questionable finding. All the confirmed erroneous results were recorded in error log.

\section{Protocol for repeat test}

If a laboratory error is suspected after analysis of the reported data, clinician forward and order request marked as "Confirmation Request for (test) of (ID No., date and time). The request includes the physician's name and cell number. Upon receipt of a confirmation request, the laboratory

a. Checks original data to detect transcription errors;

b. Repeats study on original specimen (if available) to detect error in original determination;

c. Repeats determination on a new specimen to detect any possible error in patient or specimen identification;

d. If steps (b) and (c) confirm the original results, the clinical findings and drug history are reviewed for possible unsuspected disease or complications and for possible drug interferences;

e. The results of the confirmation are reported to the physician by the clinical chemistry specialist/ technologist (no charge is made to the patient for a Confirmation Request).

\section{Results:}

Among a total of 32000 test analyses, clinicians and laboratory consultant notified a total 113 errors which were confirmed as laboratory mistakes; this was a relative frequency of $0.35 \%$. The distribution of the 
total errors was: preanalytical $20.35 \%$, analytical $12.39 \%$, and postanalytical $67.26 \%$ (Table I). The preanalytical phase was found to have the second highest number of errors. Causes of preanalytical errors are shown in Table II. The most frequent problems were from drawing of blood distal to infusion line so that specimens became diluted with an intravenous infusion solution. This problem contributed near about $40 \%$ errors in preanalytical phase. The other frequent mistakes were specimen collected in the wrong type of collection tubes, in particular, incorrect blood anticoagulant (21.7\%), long tourniquet time (8.7\%), underfilling of blood collection tube (8.7\%) and wrong demographic data (21.7\%).

\section{Table-I}

\begin{tabular}{lcc}
\multicolumn{3}{c}{ Frequency of laboratory errors } \\
Types of errors & Number & Percentage \\
\hline Preanalytical & 23 & 20.35 \\
Analytical & 14 & 12.39 \\
Postanalytical & 76 & 67.26 \\
\hline Total & 113 & 100 \\
\hline
\end{tabular}

Table-II

\section{Causes of preanalytical errors}

\begin{tabular}{lcc} 
Causes of errors & Number & Percentage \\
\hline $\begin{array}{l}\text { Specimen drawn from distal } \\
\text { to IV infusion }\end{array}$ & 9 & 39.2 \\
$\begin{array}{l}\text { Contaminated specimen for } \mathrm{K}^{+}, \\
\text {first put into GREY tube and } \\
\text { then to GREEN tube }\end{array}$ & 5 & 21.7 \\
$\begin{array}{l}\text { Long tourniquet time } \\
\begin{array}{l}\text { Underfilling of blood collection } \\
\text { tube }\end{array}\end{array}$ & 2 & 8.7 \\
$\begin{array}{l}\text { Wrong ID } \\
\text { Total }\end{array}$ & 5 & 23 \\
\hline
\end{tabular}

In the analytical phase, we identified total 14 errors, accounting for $12.39 \%$ of the total errors. We divide these analytical errors into 2 broad categories \% random errors (any random deviation from the laboratory mean) contributed $71.4 \%$ of analytical errors and remaining $28.6 \%$ were identified as systematic errors (a trend or shift away from the laboratory mean) (Table III).

In the postanalytical phase, the highest number of errors was observed, accounting for $67.26 \%$ of the total errors. Causes of postanalytical errors are shown in Table IV. Most of the errors (52 out of 76) were due to transcription errors. In another 14 cases, problems in within-laboratory communication delayed the results, and an excessive turn around time (TAT) was recorded in these cases. Because of improved information procedures, failure to report critical values was found in another 10 cases. However, new types of errors have emerged, particularly those attributable to the staff's application of new information procedures.

\section{Table-III}

Causes of analytical errors

\begin{tabular}{lcc} 
Causes of errors & Number & Percentage \\
\hline Random errors & 10 & 71.4 \\
Systematic errors & 4 & 28.6 \\
\hline Total & 14 & 100 \\
\hline
\end{tabular}

Table-IV

Causes of postanalytical errors

\begin{tabular}{lcc} 
Causes of errors & Number & Percentage \\
\hline $\begin{array}{l}\text { Transcriptional errors } \\
\text { (most common) }\end{array}$ & 52 & 68.4 \\
Turn around time failure & 14 & 18.4 \\
Failure to report critical values & 10 & 13.2 \\
\hline Total & 76 & 100 \\
\hline
\end{tabular}

\section{Discussion:}

Many diagnostic errors are associated with laboratory testing, and many of these are preventable. However, a reduction in testing-related diagnostic errors (TDE) is hindered by the absence of a well-defined relationship between diagnostic harm and the testing process (whether from laboratory or nonlaboratory sources) as well as by a lack of relevant measures for evaluation ${ }^{7}$. Advances in technology have also contributed to the increased importance of laboratory tests. In the past, 
laboratory tests were used to identify organ and system dysfunctions or diseases. While this is still true, testing now-a-days is used to diagnose disease subtypes, as occurs when pathology reports of cancer are accompanied by tumour-specific and patient-specific molecular analyses, data which help physicians determine optimum therapies and a patient's likely response to treatment ${ }^{8,9}$.

However, in this study our findings show that, at present, the most frequent source of erroneous results in the clinical laboratories seems to be in the preanalytical and postanalytical steps in the testing process, with fewer mistakes occurring during the actual analytical step. These findings are in consistence with the findings of other researchers in this field ${ }^{2-4,10}$. Today, the quality system for clinical laboratories must include promotion of accuracy in the analytical phase as well as the assurance of the reliability of preanalytical and postanalytical activities.

In the present study, most of the errors were found in preanalytical and postanalytical phase, These findings are consistent with the findings of some other studies.

Our findings of a large percentage of laboratory mistakes occurring in the preanalytical and postanalytical phases indicate that the active monitoring of all potential defects calls for the assistance of non-laboratory personnel, to enable the inclusion of steps outside the laboratory. The greatest quantitative reductions in laboratory errors are likely to be achieved through interdepartmental cooperation designed to improve the quality of specimen collection and data dissemination ${ }^{10}$. Cooperation with clinicians and personnel outside the laboratory is therefore the key to improvement, and a clinical audit is an important component in the quality assurance system for the clinical laboratories. Total compliance with ISO 15189 with special emphasis on documentation should allow us to achieve a consensus on laboratory error and on the implementation and reviewing corrective and preventing measures at regular interval.

\section{References:}

1. Dighe MS, Marker RS, Lewandrowski KB. Medicolegal liability in laboratory medicine. Semin Diagn Pathol 2007; 24(2): 98"107.

2. Ross JW, Boone DJ. Assessing the effect of mistakes in the total testing process on the quality of patient care [Abstract 102]. In: Martin L, Wagner W, Essien JDK, eds. 1989 Institute of Critical Issues in Health Laboratory Practice. Minneapolis, MN: DuPont Press, 1991.

3. Bachner P, Boone DJ, Herron R, Howanitz PJ, Meier F, Schifman R, Zarbo RJ. College of American Pathologists Outcomes Working Group, blood bank quality assurance questionnaire. Northfield, IL: CAP, 1991.

4. Boone J, Steindel SD, Herron R, Howanitz PJ, Bachner P, Meier F, Schifman RB, Zarbo RB. Transfusion medicine monitoring practices. Arch Pathol Lab Med 1995; 119: 999-1006.

5. Plebani M. Errors in clinical laboratories or errors in laboratory medicine. Clin Chem Lab Med 2006; 44(6):750-759.

6. Forsman RW. Why is the laboratory an afterthought for managed care organizations? Clin Chem 1996; 42: 813"816.

7. Paul L Epner, Janet E Gans, Mark L Graber. When diagnostic testing leads to harm: a new outcomes-based approach for laboratory medicine. BMJ Qual Saf 2013; 22:ii6-ii10.

8. Igbokwe A, Lopez-Terrada DH. Molecular testing of solid tumors. Arch Pathol Lab Med 2011;135:67-82.

9. Mehta S, Shelling A, Muthukaruppan A, et al. Predictive and prognostic molecular markers for cancer medicine. Ther Adv Med Oncol. 2010;2:125-48.

10. Mario Plebani. The detection and prevention of errors in laboratory medicine. Annals of Clinical Biochemistry 2010; 47: $101-110$

11. Blumenthal D. The errors of our ways. Clin Chem 1997; 43:1305. 